\title{
The Use of Model-based Iterative Reconstruction to Optimize \\ Chest CT Examinations for Diagnosing Lung Metastases in \\ Patients with Sarcoma : A Phantom Study
}

Kaasalainen, Touko

2019-01

Kaasalainen, T , Mäkelä , T , Kelaranta , A \& Kortesniemi , M 2019 , ' The Use of Model-based Iterative Reconstruction to Optimize Chest CT Examinations for Diagnosing Lung Metastases in Patients with Sarcoma : A Phantom Study ', Academic Radiology, vol. 26 , no. 1 , pp. 50-61 . https://doi.org/10.1016/j.acra.2018.03.028

http://hdl.handle.net/10138/310694

https://doi.org/10.1016/j.acra.2018.03.028

publishedVersion

Downloaded from Helda, University of Helsinki institutional repository.

This is an electronic reprint of the original article.

This reprint may differ from the original in pagination and typographic detail.

Please cite the original version. 


\section{Original Investigation}

\section{The Use of Model-based Iterative Reconstruction to Optimize Chest CT Examinations for Diagnosing Lung Metastases in Patients with Sarcoma: A Phantom Study}

Touko Kaasalainen, PhD, Teemu Mäkelä, PhLic, Anna Kelaranta, PhD, Mika Kortesniemi, PhD

Rationale and Objectives: This phantom study aimed to evaluate low-dose (LD) chest computed tomography (CT) protocols using model-based iterative reconstruction (MBIR) for diagnosing lung metastases in patients with sarcoma.

Materials and Methods: An adult female anthropomorphic phantom was scanned with a 64-slice CT using four LD protocols and a standard-dose protocol. Absorbed organ doses were measured with 10 metal-oxide-semiconductor field-effect transistor dosimeters. Furthermore, Monte Carlo simulations were performed to estimate organ and effective doses. Image quality in terms of image noise, contrast, and resolution was measured from the CT images reconstructed with conventional filtered back projection, adaptive statistical iterative reconstruction, and MBIR algorithms. All the results were compared to the performance of the standard-dose protocol.

Results: Mean absorbed organ and effective doses were reduced by approximately $95 \%$ with the LD protocol (100-kVp tube voltage and a fixed 10-mA tube current) compared to the standard-dose protocol (120-kVp tube voltage and tube current modulation) while yielding an acceptable image quality for diagnosing round-shaped lung metastases. The effective doses ranged from 0.16 to $2.83 \mathrm{mSv}$ in the studied protocols. The image noise, contrast, and resolution were maintained or improved when comparing the image quality of LD protocols using MBIR to the performance of the standard-dose chest CT protocol using filtered back projection. The small round-shaped lung metastases were delineated at levels comparable to the used protocols.

Conclusions: Radiation exposure in patients can be reduced significantly by using LD chest CT protocols and MBIR algorithm while maintaining image quality for detecting round-shaped lung metastases.

Key Words: Low-dose chest CT; model-based iterative reconstruction; Monte Carlo simulation; MOSFET measurements; soft tissue sarcoma. (C) 2018 The Association of University Radiologists. Published by Elsevier Inc. All rights reserved.

\section{INTRODUCTION}

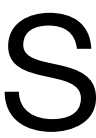

oft tissue sarcoma is a cancer that originates in the soft tissues of the body, such as muscles, tendons, ligaments, cartilage, fat tissue, lymph and blood vessels, or nerves. Tumors are often located in the limbs, head and neck, chest, or abdomen; the lungs are the most common site

\section{Acad Radiol 2019; 26:50-61}

From the HUS Medical Imaging Center, Radiology, University of Helsinki and Helsinki University Hospital, P.O. Box 340, Helsinki FI-00290, Finland (T.K., T.M., A.K., M.K.); Department of Physics, University of Helsinki, Helsinki, Finland (T.M., M.K.). Received February 16, 2018; revised March 23, 2018; accepted March 29, 2018. Funding: This study was supported by the State Subsidy for University Hospitals in Finland and a research grant from the Radiological Society of Finland. Address correspondence to: T.K. e-mail: touko.kaasalainen@hus.fi

(C) 2018 The Association of University Radiologists. Published by Elsevier Inc. All rights reserved.

https://doi.org/10.1016/j.acra.2018.03.028 of metastatic disease in soft tissue sarcoma $(1,2)$. Therefore, patients at high risk of metastases are usually evaluated with chest computed tomography (CT) scans that can typically show round-shaped sarcoma metastases. Dose reduction in CT has become a major objective in optimizing radiological examinations. This finding is due to increased CT use in diagnosing diseases in the chest and other body areas and CT's significant role in the accumulated radiation dose of the population. In accordance with current knowledge, the likelihood of presenting with stochastic adverse effects (eg, cancer) is assumed to increase linearly with radiation dose $(3-5)$.

According to the commonly accepted ALARA (as low as reasonably achievable) principle, examinations using ionizing radiation should be performed with a radiation dose that is as low as reasonably achievable while maintaining sufficient image quality for diagnosis. Optimization strategies for chest CT include the use of automatic tube current modulation (TCM), 
lowered tube voltage, adaptive beam collimation in helical scans, and partial scanning (organ dose modulation); these strategies have all been used to reduce radiation exposure to tissues and to optimize image quality $(6-10)$. One of the most promising CT optimization techniques is the continuously developing iterative reconstruction algorithms that aim to overcome the limitations of the traditional reconstruction method of filtered back projection (FBP) for image quality and diagnostic dose efficiency. The iterative reconstruction algorithms include statistical (hybrid) and model-based iterative reconstruction (MBIR) techniques. The former only models the noise statistics, whereas the latter uses a more complex system of prediction models, including modeling of optical factors, such as $\mathrm{x}$-ray tube and detector responses, in addition to voxel projections, $\mathrm{x}$-ray beam spectra, and noise statistics (11). Several studies have reported significant dose reduction capabilities of iterative reconstruction methods (especially with MBIR) in the chest and other body areas while maintaining or improving diagnostic image quality (12-31). However, some researchers have warned about the possibilities of missing clinically significant lesions with lowdose (LD) abdominal and chest CT protocols using iterative reconstruction techniques $(32,33)$. The image noise magnitude and texture of the iteratively reconstructed images depend on the scanned tissue, and noise magnitude may behave differently at tissue boundaries compared to uniform regions (34).

Our study aimed to evaluate LD chest CT protocols for diagnosing metastases in patients with sarcoma by determining absorbed radiation doses with an anthropomorphic phantom and metal-oxide-semiconductor field-effect transistor (MOSFET) dosimeters. The aim was also to provide three-dimensional (3D) dose assessment by performing voxel-based Monte Carlo (MC) simulations to determine organ and effective doses produced by each scanning protocol to the anthropomorphic model. This approach was selected to achieve a comprehensive dose assessment with the volumetric dose distribution offered by MC simulations applied in conjunction with point-dose measurements. Finally, to provide the image-quality aspect of optimization, we compared the image qualities of the images reconstructed with FBP, adaptive statistical iterative reconstruction (ASIR), and MBIR algorithms.

\section{MATERIALS AND METHODS}

\section{Dose Measurements and Simulations}

An adult female anthropomorphic phantom (ATOM Model 702-D; CIRS, Norfolk, VA) was scanned in a supine position with a 64-slice CT scanner (GE Discovery CT750 HD; GE Healthcare, Milwaukee, WI). Four LD protocols and a standard-dose protocol were used to delineate the lung metastases in the patient with sarcoma in the anthropomorphic phantom. The anatomic characteristics of the female phantom were $55 \mathrm{~kg}$ (weight), $160 \mathrm{~cm}$ (height), and $20 \times 25 \mathrm{~cm}$ (chest dimensions). The phantom was made of tissue-equivalent epoxy resins with the following physical densities: $1.60 \mathrm{~g} / \mathrm{cm}^{3}$ (bone tissue), $1.05 \mathrm{~g} / \mathrm{cm}^{3}$ (soft tissue), $1.07 \mathrm{~g} / \mathrm{cm}^{3}$ (spinal cord), $1.15 \mathrm{~g} / \mathrm{cm}^{3}$ (spinal disks), and $0.21 \mathrm{~g} / \mathrm{cm}^{3}$ (lungs). The phantom was set to the CT scanner isocenter by lasers and visual landmarks on the phantom. The scan range was set according to a typical chest CT examination (from the apex of the lungs to the lateral phrenic angles). The scanning parameters and dose indices (volume computed tomography dose index $\left[\mathrm{CTDI}_{\mathrm{vol}}\right.$ and dose-length product [DLP]) are shown in Table 1. The reported GE noise indices (NIs) are relative to the target image noise described as the standard deviation of CT numbers in Hounsfield units. The TCM in GE CT systems is calculated from the last scout image (no matter how many scout images are scanned) and the selected NI; increasing the NI lowers the total dose. The following were used in each helical CT scanning protocol: 0.984 pitch, $8 \times 5 \mathrm{~mm}$ detector configuration, and a large scan field of view.

Before dose measurements, 10 high-sensitivity TN1002RD MOSFET dosimeters (Best Medical, Canada) with high-bias settings were calibrated in air for 100- and 120$\mathrm{kVp}$ tube voltages with clinical CT beam settings by using a calibrated $10-\mathrm{cm}$ pencil ionizing chamber (RaySafe Xi; Unfors RaySafe AB, Billdal, Sweden). After calibration, the dosimeters were inserted to the adult female phantom, and organ doses to the lungs (four dosimeters), liver (three dosimeters), thyroid gland, heart, and right breast (one dosimeter each) were measured (Fig 1). The scans using LD protocols were repeated 50 times before reading the dosimeters. This procedure was performed to improve the reproducibility of the dose measurements and to achieve sufficiently high radiation doses in the dosimeters. This method was repeated three times (or total of 150 scans). The scans using standard-dose protocol were repeated three times before reading the dosimeters to provide similar reproducibility compared to the LD exposures. This method was repeated three times (or a total of nine scans). The individual dosimeter readings were used to estimate the measurement uncertainty (random error) of the mean absorbed point doses.

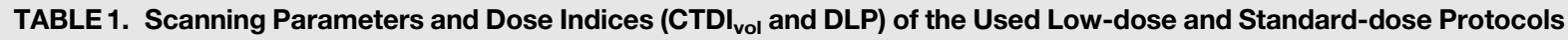

\begin{tabular}{lllcrr} 
Protocol & Tube Voltage $(\mathrm{kVp})$ & Tube Current/GE NI & Rotation Time $(\mathrm{s})$ & CTDI $_{\text {vol }}(\mathrm{mGy})$ & DLP $(\mathrm{mGy} \cdot \mathrm{cm})$ \\
\hline Low dose 1 & 100 & Fixed 10 mA & 0.4 & 0.20 & 6.33 \\
Low dose 2 & 100 & $\mathrm{NI}=50$ & 0.4 & 0.31 & 9.91 \\
Low dose 3 & 120 & Fixed 10 mA & 0.4 & 0.31 & 10.05 \\
Low dose 4 & 120 & $\mathrm{NI}=40$ & 0.4 & 0.49 & 15.70 \\
Standard dose & 120 & $\mathrm{NI}=15$ & 0.5 & 3.58 & 115.08 \\
\hline
\end{tabular}

$\mathrm{CTDI}_{\mathrm{vol}}$, volume computed tomography dose index, DLP, dose-length product; NI, noise index. 


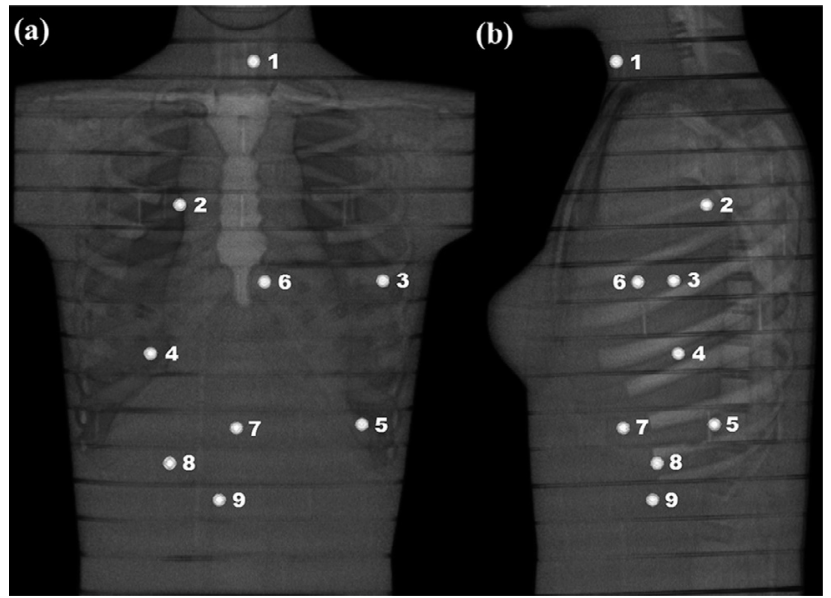

Figure 1. Metal-oxide-semiconductor field-effect transistor positions inside the anthropomorphic phantom shown in anterior-posterior (a) and lateral (b) directions. The locations were thyroid (1), lungs $(2-5)$, heart (6), and liver (7-9). Additionally, one metal-oxide-semiconductor field-effect transistor dosimeter was positioned on the right breast (not visible in the images).

To estimate organ and effective doses, MC simulations (ImpactMC; Vamp GmbH, Germany) were performed, along with the point doses measured with MOSFET dosimeters. MC simulations were done using $3 \mathrm{D}$ voxelized data that were derived from previously acquired CT images of the whole anthropomorphic phantom. To enable simulations with existing computer memory capacity, a slice thickness of $2.5 \mathrm{~mm}$ was used in the simulations. As a prerequisite for the MC simulations, the x-ray spectrum was simulated for both 100- and 120$\mathrm{kVp}$ tube voltages using the Spekcalc 2.0 program (35) and information from the vendor specifications (tube type, halfvalue layer, anode material, and filtration). Furthermore, beam dose profile free-in-air was measured with the same pencil ionization chamber as used in the MOSFET calibration to provide source data for bowtie filter thickness across the axial scan plane for the large body scan field of view. The method used to define the shape of the bowtie filter corresponded to the previously published static x-ray tube method (36). Bowtie thickness data and simulated spectrum were then used as input for the MC simulation, together with the anthropomorphic phantom CT voxel data, scan range, pitch, and tube current values based on the TCM data. The TCM accounted for tube current $\mathrm{z}-$ axis variation and angular range as reported by the $\mathrm{CT}$ scanner. Two simulations were performed for each scan setting with the x-ray tube rotational start angles at $0^{\circ}$ and $180^{\circ}$ to acquire complementary dose data for the uncertainty evaluation of the simulated results (to manage uncertainty contribution due to varying tube start angles during the scan). Absorbed-dose voxel maps were acquired as the output of simulations. Spherical volumes of interest (VOIs) with 5-mm radii were placed at the corresponding MOSFET dosimeter locations, and the mean doses were recorded to be used in comparison to the experimental MOSFET dosimetry point-dose results.

Radiation-sensitive organs and tissues were segmented from the anthropomorphic phantom to determine mean organ doses based on the whole segmented organ volumes in the simulated 3D dose maps. The brain, breasts, lungs, bones, spinal cord, cartilage, and air were segmented based on Hounsfield unitlevel thresholding and manual identification. Skin was determined as the surface layer on the phantom. Other radiosensitive organs were brought from the International Commission on Radiological Protection (ICRP) reference adult female computational phantom (37) by coregistering the voxel model with ATOM phantom CT data. Three regions (the head and neck, the pelvis, and the remainder of the torso) were matched separately using rigid registration. Organs were adjusted to accommodate appropriate soft tissue regions. No overlap was allowed. As our phantom lacked physical legs, arms, and shoulders, these were also omitted from the segmentations. Finally, the organ segmentations were dilated to match the ICRP reference volumes apart from bone, lung, brain, and breast tissues that were physically defined in the anthropomorphic phantom. Organs defined as a separate wall and contents (such as stomach) were combined into single segments.

We compared the absorbed radiation doses measured with MOSFET dosimeters and compared organ doses and effective doses (determined by MC simulations) in each LD helical chest CT protocol to those of the standard-dose protocol. We also compared the point organ doses that resulted from the MOSFET measurements and $\mathrm{MC}$ simulations to each other. Furthermore, we determined the conversion coefficients from the DLP to the effective dose to be used for our anthropomorphic phantom.

\section{Image Quality Measurements and Analysis}

In addition to the irradiations performed for the dose measurements, we also performed separate image quality scans with the same scanning parameters (Table 1). Each scan was repeated twice. MOSFET dosimeters were removed from the anthropomorphic phantom to minimize image artifacts caused by the dosimeter and wire structures. Three round-shaped soft tissue plugs ( $5 \mathrm{~mm}$ in diameter and $25 \mathrm{~mm}$ long) were inserted into the lungs, and three lung holes were left empty ("air plugs") to simulate metastatic lesions. The images were reconstructed with the following four reconstruction methods: FBP with a standard kernel, ASIR (40\% level of ASIR-FBP blending), standard and chest kernels, and MBIR (VEO, GE Healthcare). The standard reconstruction kernel is typically used in routine examinations, whereas the chest kernel is specifically designed for mediastinum and lung details. The slice thickness was $0.625 \mathrm{~mm}$, the image matrix was $512 \times 512$, and the display field of view was $28 \mathrm{~cm}$ for all the series. This resulted in a $0.547 \times 0.547 \times 0.625 \mathrm{~mm}^{3}$ voxel size. We compared the image resolution, noise, and contrast in each LD setting and reconstruction to those of the standard-dose images. Image quality analysis steps (shown in Fig 2 and described further) were implemented in MATLAB (MathWorks, Natick, MA).

Image noise magnitude was measured by the following two methods: subtracting the repeated image quality scans, and fitting Gaussian distributions to VOIs and dividing the resulting standard deviations by $\sqrt{ } 2$ (soft tissue) or fitting a 
truncated Gaussian distribution to a combined histogram of the two scans and recording the standard deviations (lung). The fitting was performed by the simplex search method implemented in MATLAB (38). The former subtraction approach removes systematic inhomogeneity in the phantom materials. The latter approach was required because the scanner did not record CT values correctly below $-1000 \mathrm{HU}$ (Fig 2c). The values below the cutoff were omitted from the fitting. The spherical VOIs (diameter $15 \mathrm{~mm}$ ) were placed manually with the aim of filling the available space. The manual placing was chosen to avoid support structures, tissue boundaries, air gaps between phantom slabs, plug inserts, the edges of the field of view, or the scan range and other possible nonuniform phantom regions. The total numbers of VOIs were 99 (soft tissue) and 87 (lung). Defining noise by histogram fitting has been used previously by Kaasalainen et al. (39).

Image contrast measures were defined as (1) the expectation values of the Gaussian fits obtained in the image noise step mentioned earlier (for lung), (2) the average of the VOI means (soft tissue), or (3) taking the mean Hounsfield unit value from the inserted tissue or air plugs.

Image noise textures were evaluated by calculating twodimensional noise power spectrum (NPS (40)) from the

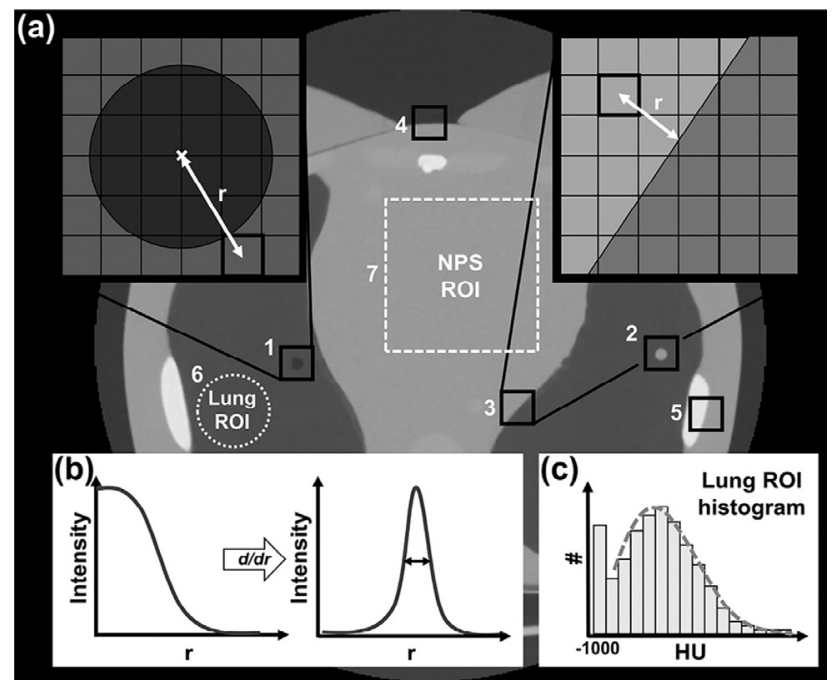

Figure 2. (a) A schematic representation of image quality analysis. Resolution was assessed by two methods, radial averaging air (1) or soft tissue (2) plugs in the lung (top left) and averaging by the distance perpendicular to a tissue border ( $3-5$, top right). The pixel intensities are sorted by the distance from the plug center or tissue boundary, $r$ (double-headed arrows), and the resulting oversampled edge-spread function is differentiated to produce the line spread function (b). The full width at half maxima of the resulting line spread function is recorded as the resolution value. (c) Noise and contrast in the lung (6) are evaluated by fitting Gaussian distributions to the truncated histograms. Hounsfield unit values below -1000 in the first histogram bin are omitted because of saturation. The fitted standard deviation and expectation values are recorded as the noise and contrast measures. Not shown in the image are the actual ROls defined for lung and soft tissues. NPSs are calculated from a square region (7) in the mediastinum and averaged over multiple slices. NPS, noise power spectrum; $\mathrm{ROI}$, region of interest. subtraction images using $101 \times 101$ pixel regions of interest in 92 slices in the mediastinum. The obtained two-dimensional NPSs were first averaged over the slices and then radially averaged using a $0.25 \mathrm{lp} / \mathrm{cm}$ window to produce onedimensional representations. These were normalized to unity at the peak power and were compared qualitatively.

Two complementary methods were used to measure image resolution (upper corners in Fig 2a and b). First, edge spreading was estimated at different tissue boundaries (air vs soft tissue, soft vs lung tissue, and soft vs bone tissue). We selected 20 sites for each boundary type and examined profiles perpendicular to the boundary to provide oversampled edge-spread values. Primary component analysis was used to fit planes at the sites' local neighborhoods to provide the edge normals. Voxel Hounsfield unit values and normal distances were recorded and two partial error functions were fitted in the least squares sense: the first and the second parts of the edge were allowed to be described by two independent error functions with the constraining requirement of smoothness at the intersection. The approach is somewhat similar to that of Sanders et al. (41). The combined fit functions were differentiated and the full widths at half maxima (FWHMs) of the resulting line spread functions were used as resolution measures. Second, a corresponding analysis was performed for the seven tissue or air plugs inserted into the phantom lung tissue. Voxel values were recorded with respect to the distance from the plug central axis. The axis midpoints were obtained by the in-plane centers of mass of the plug neighborhoods after thresholding by the Otsu method (42). The oversampled edge-spread functions and FWHMs were obtained in the same manner as previously discussed after radially averaging the profiles from multiple slices. The latter "cylinder" approach was previously applied, for example, by Suomalainen et al. (43). In both cases, a 0.1-mm running average was used in obtaining the prefitting distanceHounsfield unit profiles. All the edge fitting functions were visually verified to match the underlying data.

\section{RESULTS}

\section{Organ Doses and Effective Doses}

Radiation dose in terms of CTDI ${ }_{\mathrm{vol}}$ and DLP values of the LD protocol using a $100-\mathrm{kVp}$ tube voltage and a $10-\mathrm{mA}$ tube current (LD 1) was approximately 95\% lower than that of the standard-dose protocol. Figure 3 shows the measured and simulated mean absorbed organ doses in each LD and standard-dose protocol. The organ doses determined with the voxel-based MC simulations corresponded well with those measured from the same points of the female phantom with MOSFET dosimeters. On average, the MC simulations systematically resulted in $10 \%$ $(7 \%-14 \%)$ lower absorbed organ doses than the MOSFET measurements. The lowest organ doses were achieved with the LD 1 protocol, whereas the highest doses were measured with the standard-dose protocol $(120 \mathrm{kVp}$ and NI of 15). According to the MOSFET measurements, the highest mean absorbed organ doses in each protocol were observed in the thyroid 


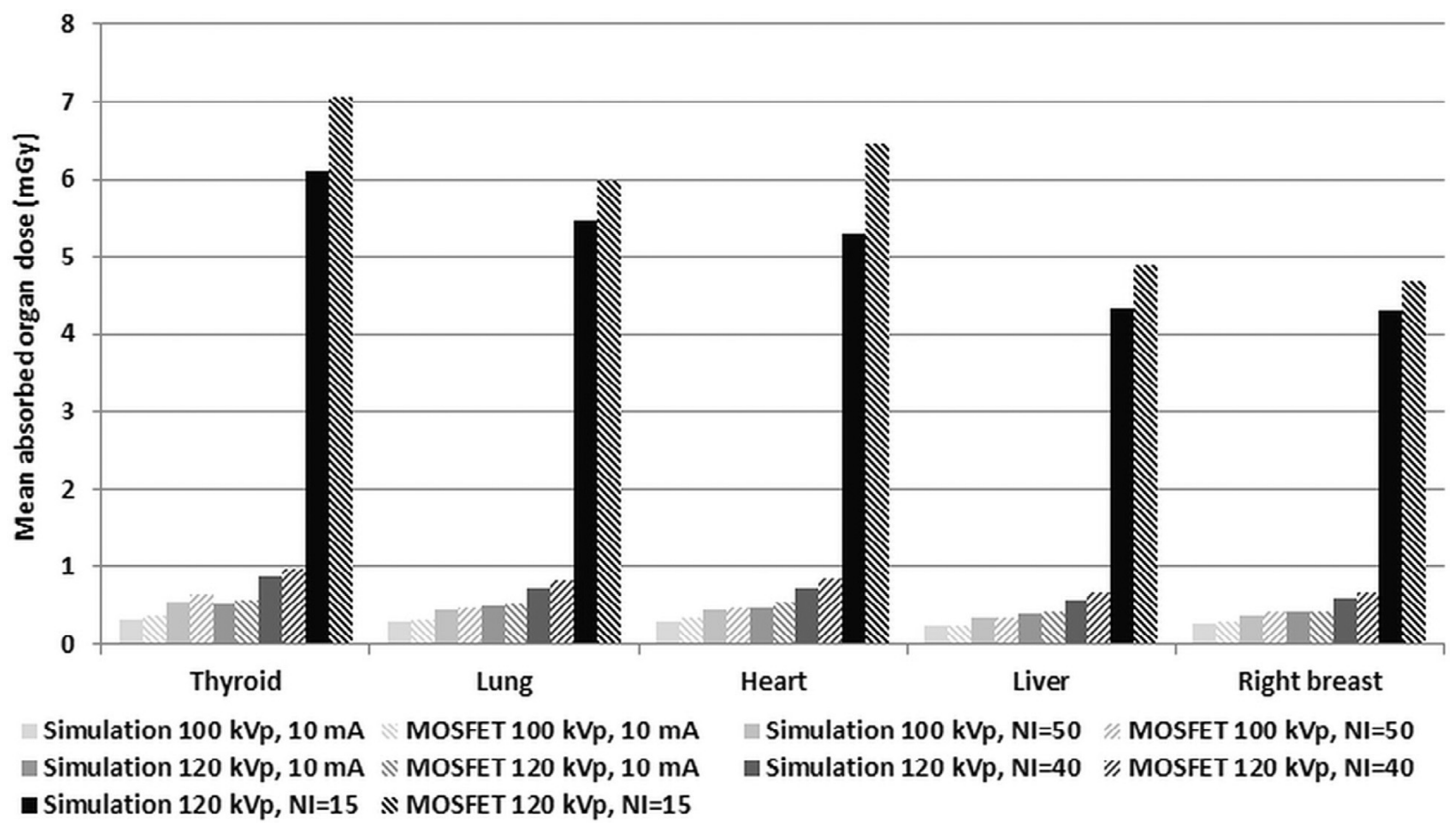

Figure 3. Mean absorbed organ doses produced by different scanning protocols. The doses were determined from the same organ locations in the Monte Carlo simulations and MOSFET measurements. MOSFET, metal-oxide-semiconductor field-effect transistor; NI, noise index.

gland (from $0.37 \pm 0.02$ to $7.07 \pm 0.32 \mathrm{mGy}$ ), and the lowest mean organ doses were observed in the liver (from $0.24 \pm 0.01$ to $4.89 \pm 0.24 \mathrm{mGy})$. As the scanning range ended in the liver and two high-sensitivity MOSFET dosimeters in the liver were positioned outside the primary scanning range, the absorbed doses measured inside the liver varied from 0.17 to $0.30 \mathrm{mGy}$ and from 3.54 to $5.77 \mathrm{mGy}$ with the LD 1 protocol and the standard-dose protocol, respectively. According to both MOSFET measurements and MC simulations, the LD 1 protocol resulted in an approximately $95 \%$ decrease in the mean absorbed organ doses compared to the standard-dose protocol, whereas the LD 4 protocol (using $120 \mathrm{kVp}$ and NI of 40) resulted in approximately $86 \%$ reduced doses. The measured relative organ dose savings agree with the differences in the $\mathrm{CTDI}_{\mathrm{vol}}$ and DLP values of the protocols.

The organ doses in different tissues together with the effective doses estimated in the anthropomorphic model with MC simulations for each scanning protocol are shown in Table 2. According to the simulations, the absorbed organ doses were the highest in organs located inside or in the near vicinity of the primary beam (thymus, lungs, breasts, esophagus, heart, thyroid, liver, spleen, stomach, pancreas, and gallbladder). The mean conversion coefficient from the scanner reported DLP values to the effective dose, $k$, was calculated for our anthropomorphic phantom $(k=0.024 \mathrm{mSv} / \mathrm{mGy} \cdot \mathrm{cm})$.

The simulated dose maps for the standard-dose and LD 1 helical scans are presented in Figure 4 as comparative coronal midplane representations. The presented dose maps were simulated with an $\mathrm{x}$-ray tube start angle of $0^{\circ}$ (thus, the $\mathrm{x}$-ray tube was positioned on the anterior side of the anthropomorphic phantom at the beginning of the simulated scan). The x-ray tube start angle affected the organ doses the most in tissues irradiated only partially. Smaller variations were seen for tissues located wholly inside the primary scanning range. For example, the lungs and the heart received absorbed organ doses that were within $2 \%$ between the scans performed with $0^{\circ}$ and $180^{\circ} \mathrm{x}$-ray tube start angles, whereas the absorbed doses in organs such as the liver $(\sim 3 \%)$, the thyroid gland $(\sim 5 \%)$, the pancreas $(\sim 6 \%)$, and the spleen $(\sim 9 \%)$ altered more, depending on the $\mathrm{x}$-ray tube start position.

\section{Image Quality Measurements and Analysis}

The image noise was the lowest in images reconstructed with the MBIR algorithm. The ASIR algorithm with chest reconstruction kernel resulted in the highest image noise in lung tissue; the highest image noise was observed with the FBP algorithm in soft tissue regardless of the dose level (Fig 5). The measured image noise in the MBIR images produced by the LD 1 protocol was similar to or lower than the image noise in the standard-dose images reconstructed either with the ASIR or FBP algorithms.

The image contrast remained approximately the same in lung tissue when the radiation dose was lowered (Supplementary Fig S1). However, the mean CT number value in soft tissues was somewhat decreased in the MBIR images and was more clearly decreased in the ASIR images reconstructed with the chest kernel.

The mean CT numbers increased in air plugs within the lungs regardless of the reconstruction technique when using 
TABLE 2. Organ Doses and Effective Doses Determined From the Monte Carlo Simulations for Different LD and Standard-dose Protocols

\begin{tabular}{|c|c|c|c|c|c|c|}
\hline \multirow[b]{2}{*}{ Organ/Tissue } & $\begin{array}{l}\text { ICRP } 103 \text { Tissue } \\
\text { Weighting Factor }\end{array}$ & $\begin{array}{l}\text { LD } 1(100 \mathrm{kVp} \text {, } \\
10 \mathrm{~mA})\end{array}$ & $\begin{array}{l}\text { LD } 2(100 \mathrm{kVp}), \\
\mathrm{NI}=50\end{array}$ & $\begin{array}{l}\text { LD } 3(120 \mathrm{kVp} \text {, } \\
10 \mathrm{~mA})\end{array}$ & $\begin{array}{l}\text { LD } 4(120 \mathrm{kVp}) \\
\mathrm{NI}=40\end{array}$ & $\begin{array}{l}\text { Standard Dose } \\
(120 \mathrm{kVp}), \mathrm{NI}=15\end{array}$ \\
\hline & $W_{T}$ & \multicolumn{5}{|c|}{ Equivalent Dose (mSv) } \\
\hline Active bone marrow & 0.12 & 0.0566 & 0.0882 & 0.0896 & 0.1395 & 1.0249 \\
\hline Colon & 0.12 & 0.0073 & 0.0105 & 0.0130 & 0.0188 & 0.1444 \\
\hline Lung & 0.12 & 0.2778 & 0.4270 & 0.4585 & 0.7057 & 5.1819 \\
\hline Stomach & 0.12 & 0.2632 & 0.3737 & 0.4357 & 0.6190 & 4.7250 \\
\hline Breast & 0.12 & 0.2683 & 0.3741 & 0.4316 & 0.6015 & 4.4266 \\
\hline Gonads & 0.08 & 0.0006 & 0.0009 & 0.0012 & 0.0016 & 0.0127 \\
\hline Bladder & 0.04 & 0.0012 & 0.0018 & 0.0023 & 0.0034 & 0.0259 \\
\hline Esophagus & 0.04 & 0.2572 & 0.4046 & 0.4330 & 0.6824 & 4.9770 \\
\hline Liver & 0.04 & 0.2654 & 0.3752 & 0.4379 & 0.6191 & 4.7167 \\
\hline Thyroid & 0.04 & 0.2592 & 0.4382 & 0.4146 & 0.7032 & 4.9756 \\
\hline Bone surface & 0.01 & 0.1134 & 0.1799 & 0.1799 & 0.2852 & 2.0858 \\
\hline Brain & 0.01 & 0.0038 & 0.0065 & 0.0069 & 0.0118 & 0.0838 \\
\hline Salivary glands & 0.01 & 0.0245 & 0.0418 & 0.0409 & 0.0698 & 0.5017 \\
\hline Skin & 0.01 & 0.0930 & 0.1470 & 0.1496 & 0.2361 & 1.7316 \\
\hline Adrenals & 0.0092 & 0.2245 & 0.3233 & 0.3822 & 0.5482 & 4.2295 \\
\hline Extrathoracic region & 0.0092 & 0.0208 & 0.0351 & 0.0351 & 0.0598 & 0.4231 \\
\hline Gallbladder & 0.0092 & 0.2525 & 0.3625 & 0.4218 & 0.6057 & 4.6587 \\
\hline Heart & 0.0092 & 0.2944 & 0.4521 & 0.4911 & 0.7542 & 5.5098 \\
\hline Kidneys & 0.0092 & 0.1206 & 0.1742 & 0.2044 & 0.2950 & 2.2723 \\
\hline Lymphatic nodes & 0.0092 & 0.1238 & 0.1987 & 0.2068 & 0.3313 & 2.4233 \\
\hline Muscle & 0.0092 & 0.0917 & 0.1457 & 0.1509 & 0.2396 & 1.7566 \\
\hline Oral mucosa & 0.0092 & 0.0165 & 0.0282 & 0.0288 & 0.0485 & 0.3476 \\
\hline Pancreas & 0.0092 & 0.1801 & 0.2594 & 0.3027 & 0.4365 & 3.3613 \\
\hline Small intestine & 0.0092 & 0.0223 & 0.0322 & 0.0380 & 0.0550 & 0.4236 \\
\hline Spleen & 0.0092 & 0.2408 & 0.3470 & 0.4020 & 0.5808 & 4.4665 \\
\hline Thymus & 0.0092 & 0.3119 & 0.5305 & 0.5138 & 0.8726 & 6.2617 \\
\hline Uterus & 0.0092 & 0.0007 & 0.0010 & 0.0013 & 0.0020 & 0.0149 \\
\hline Effective dose, $E$ (mSv) & $\sum W_{T}=1$ & 0.16 & 0.23 & 0.26 & 0.38 & 2.83 \\
\hline
\end{tabular}

ICRP, International Commission on Radiological Protection; LD, low dose; NI, noise index.

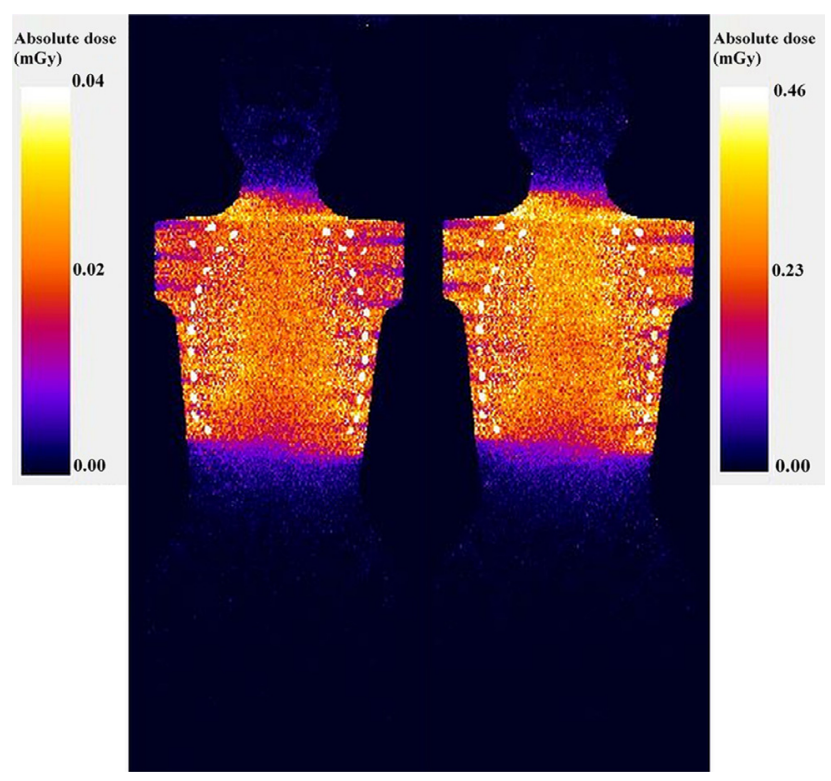

Figure 4. Simulated dose maps in the coronal plane for the low dose 1 (left) and standard-dose (right) helical scans. (Color version of figure is available online.) the LD protocols instead of the standard-dose protocol (Fig 6). The ASIR algorithm with the chest reconstruction kernel yielded the highest CT numbers in air plugs, whereas the other reconstructions resulted in similar contrast values. However, higher CT numbers were observed in the soft tissue plugs with the FBP algorithm compared to other reconstructions methods when using LD protocols.

The NPS analysis showed the image noise to be at lower spatial frequencies in the MBIR images compared to the ASIR and FBP images (Fig 7 and Supplementary Figs S2 and S3). The MBIR reconstruction algorithm had a clear dose dependency, whereas the other reconstruction algorithms did not show any variation in the normalized NPS regardless of the dose level (Supplementary Fig S2). The reconstruction kernel in the ASIR reconstructions did not affect the image noise structure in terms of the NPS. However, the FBP reconstruction algorithm yielded image noise that tended to be in higher spatial frequencies than the noise in the ASIR images. The magnitudes of the NPS in absolute scale are shown in Figure 7. The MBIR resulted in notably lower magnitudes compared to the other reconstruction algorithms. 

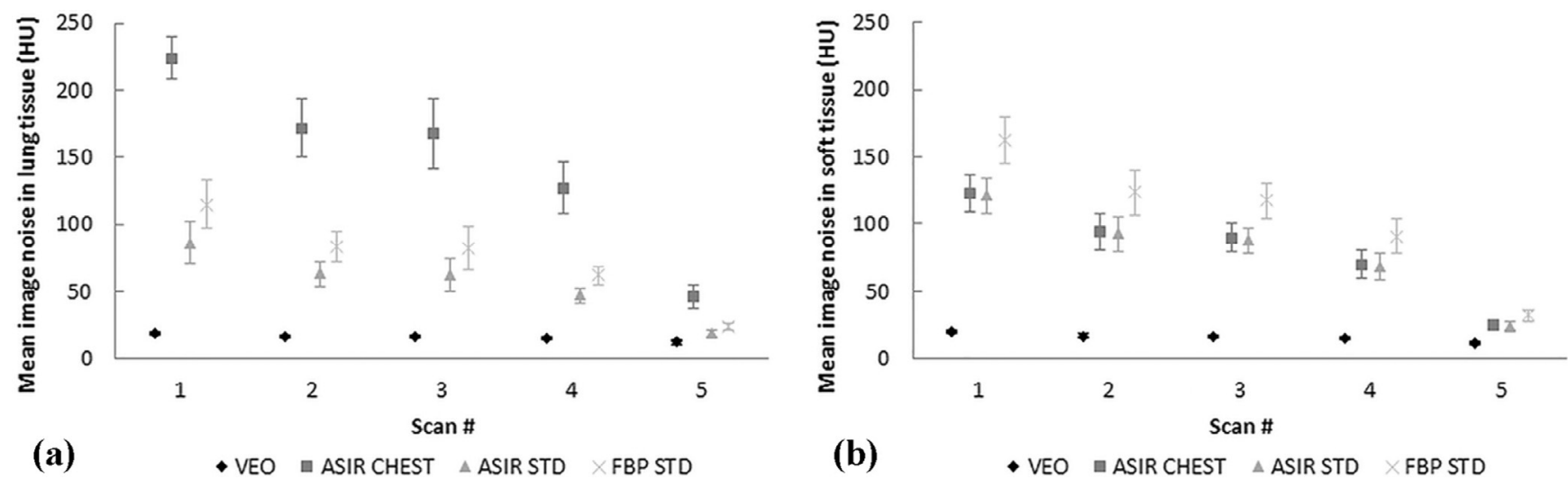

Figure 5. Mean image noise in (a) lung tissue and (b) soft tissue determined from images scanned with four low-dose protocols (scans 1-4) and a standard-dose protocol (scan 5) and reconstructed with different image reconstruction algorithms. ASIR, adaptive statistical iterative reconstruction; FBP, filtered back projection; STD, standard reconstruction kernel.

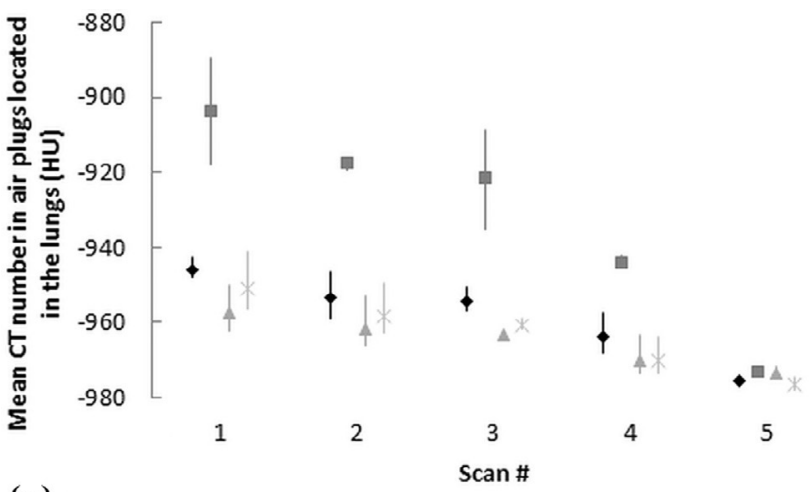

(a)

- VEO $\square$ ASIR CHEST $\triangle$ ASIR STD $\times$ FBPSTD

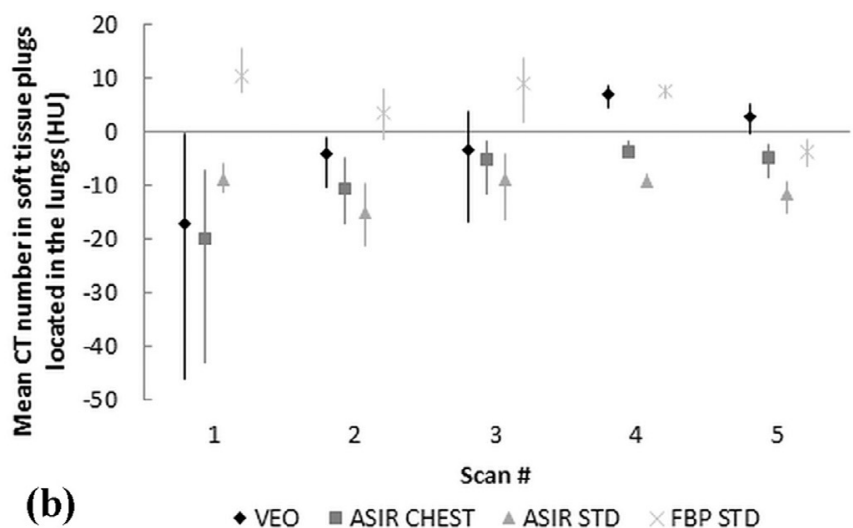

- VEO $\square$ ASIR CHEST $\quad$ ASIR STD $\times$ FBP STD

Figure 6. Mean image contrast in (a) air and (b) soft tissue plugs inserted into the phantom's lungs in chest CT images scanned with four lowdose protocols (scans 1-4) and a standard-dose protocol (scan 5) and reconstructed with different image reconstruction algorithms. ASIR, adaptive statistical iterative reconstruction; CT, computed tomography; FBP, filtered back projection; STD, standard reconstruction kernel.

The low-frequency spike especially visible in Figure $7 \mathrm{a}$ is primarily due to a slight position dependence in noise magnitude because the mediastinum regions of interest were not perfectly at the CT scanner isocenter and were surrounded by rotationally varying distributions of attenuating material (soft tissue thickness at different angles, as well as bone and lung structures).

The FWHM analysis results from tissue boundaries (ie, soft tissue vs air, soft tissue vs lung tissue, and soft tissue vs bone tissue) indicated that the MBIR algorithm yielded higher resolution than the other reconstruction algorithms (Supplementary Fig S4). While the differences between the reconstructions were the greatest in the air vs soft tissue boundaries, a clear difference was also observed in the soft tissue vs lung tissue boundaries. In the soft tissue vs bone tissue boundaries, the MBIR and FBP algorithms resulted in somewhat closer edge-spread values to each other, especially when using lower radiation exposure levels.

The edge-spread analysis performed for the soft tissue plugs inserted into the lungs indicated the same or superior performance of the MBIR algorithm compared to the other reconstruction techniques (Fig 8). Moreover, the MBIR algorithm, together with the LD 1 protocol, resulted in resolution values in the soft tissue plugs comparable to those of the FBP with the standard-dose protocol. However, the edge spreading in air plugs was higher as measured with the MBIR algorithm than that with the other reconstruction techniques most apparently because of increased noise content in FBP and ASIR images.

\section{DISCUSSION}

Chest CT scans in varying indications are one of the most common CT studies performed worldwide. Because of different needs in the required image quality, each scanning protocol should be optimized for its purpose. The aim of our phantom study was to evaluate the use of LD chest CT protocols using the MBIR algorithm for diagnosing lung metastases in patients with sarcoma. We observed dose reductions up to $95 \%$ with the constructed LD protocols while maintaining image quality at round-shaped metastases typically seen in the lungs of patients with sarcoma. Our image quality analysis results indicated MBIR to be superior compared to FBP and ASIR because of decreased image noise; MBIR also preserved image contrast at comparable levels. Additionally, our spatial resolution analysis based on edge spread showed 

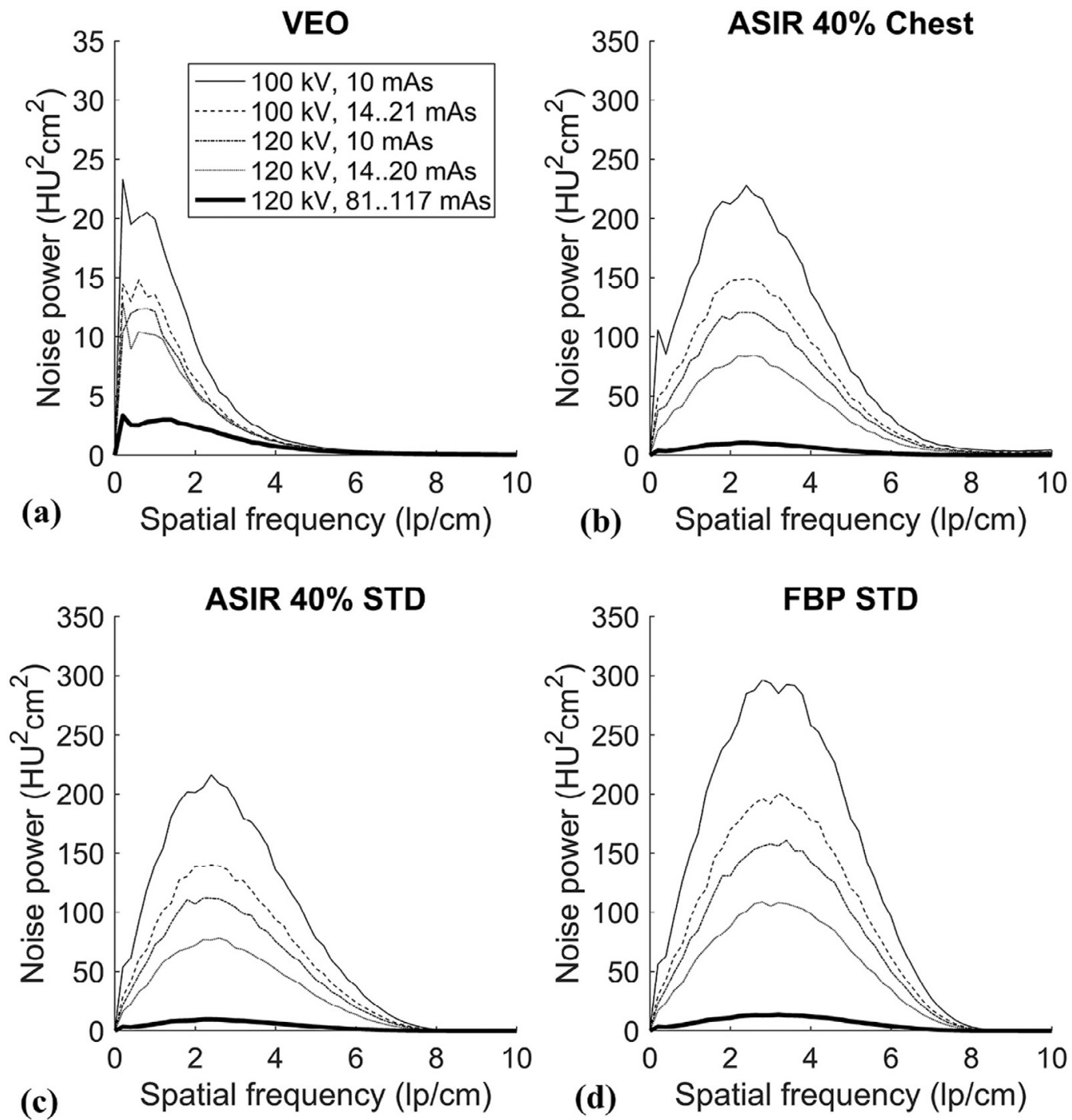

Figure 7. The noise power spectra of different image reconstruction techniques in absolute scale. The model-based iterative reconstruction algorithm (a) produced notably lower magnitudes for the NPS than the other image reconstruction algorithms (b-d). ASIR, adaptive statistical iterative reconstruction; FBP, filtered back projection; STD, standard reconstruction kernel.

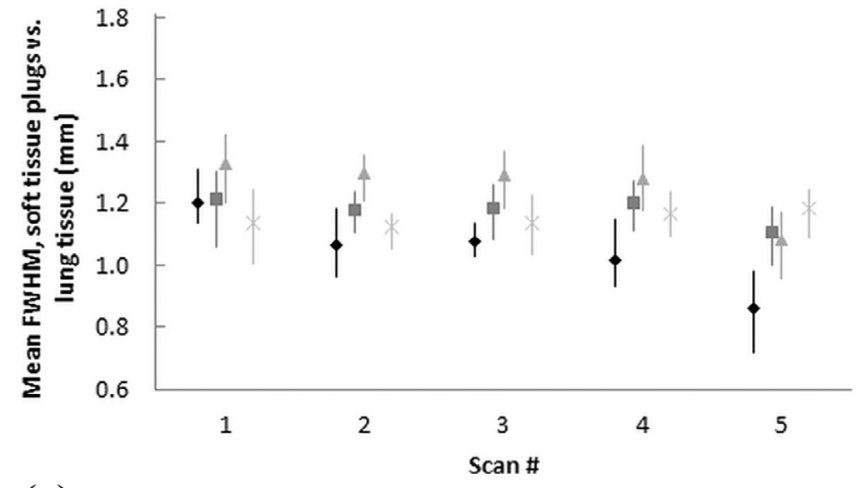

(a)

- VEO a ASIR CHEST $\triangle$ ASIR STD $\times$ FBPSTD

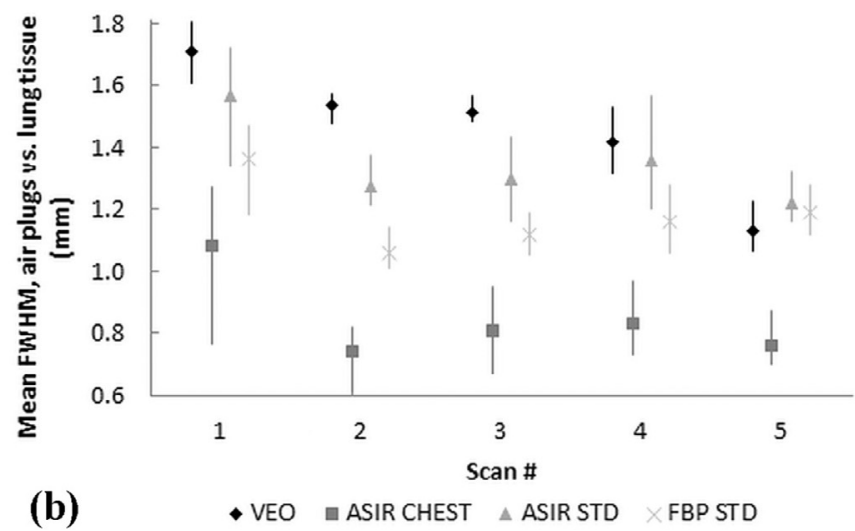

Figure 8. Edge spreading determined as the FWHM from the following tissue plug boundaries: (a) soft tissue plug vs lung tissue and (b) air plug vs lung tissue. Images were scanned with four low-dose protocols (scans 1-4) and a standard-dose protocol (scan 5) and were reconstructed with different image reconstruction algorithms. ASIR, adaptive statistical iterative reconstruction; FBP, filtered back projection; FWHM, full width at half maxima; STD, standard reconstruction kernel. 
improved or maintained spatial resolution values with MBIR compared to the FBP and ASIR algorithms.

The highest absorbed organ doses, measured with MOSFET dosimeters, were in the thyroid gland followed by (in decreasing order) the lungs, heart, breast, and liver tissue. The LD 1 protocol $(100-\mathrm{kVp}$ tube voltage and a fixed $10-\mathrm{mA}$ tube current) resulted in an approximately $95 \%$ reduction in organ doses compared to the standard-dose protocol $(120-\mathrm{kVp}$ tube voltage and GE NI of 15). The thyroid dose was the highest because of anatomy and attenuation of the neck area. The liver dose was decreased because of averaging of the measured absorbed point doses of three separate MOSFET dosimeters, in which only one dosimeter was positioned into the primary beam and two dosimeters were outside of the scanning range. Thus, the upper part of the liver was exposed to similar absorbed-dose levels as the lungs, whereas the rest of the liver was exposed to only scattered radiation. Moreover, as we used only a single MOSFET dosimeter to measure the dose in the thyroid gland and the upper part of the thyroid was outside of the primary scanning range, the uncertainty of the thyroid dose might be considerable and the actual thyroid dose could be smaller than we measured.

The MC simulations agreed with the MOSFET measurements when comparing the doses determined from the corresponding sites in the phantom. On average, the MC simulations resulted systematically in $10 \%(7 \%-14 \%)$ lower absorbed doses than the MOSFET measurements yielded. When comparing the simulated absorbed organ doses of different tissues, the highest doses were observed in tissues located in the primary scanning range or in the near vicinity, as expected. The organ doses were thus the most notable in the thymus, heart, lungs, esophagus, thyroid gland, breasts, liver, spleen, stomach, pancreas, and gallbladder. We also observed that the $\mathrm{x}$-ray tube start angle in simulations affected the organ doses the most in tissues that were irradiated only partially; lower doses were observed in tissues located wholly inside the primary scanning range because of the averaging effect. As discussed earlier, the thyroid gland was located partially outside of the primary scanning range. Therefore, the thyroid dose in the simulation was lower (from 0.26 to $4.98 \mathrm{mSv}$ with the LD 1 and standard-dose protocols, respectively) than the measured thyroid dose with MOSFET dosimeters.

Based on our simulations, the effective doses ranged from 0.16 to $2.83 \mathrm{mSv}$ in the scan protocols used. The dose level of the LD 1 protocol was comparable to the dose level of a conventional chest $\mathrm{x}$-ray study, which was reported to be in the range of $0.02 \mathrm{mSv}$ (for a posterior-anterior projection study) to $0.1 \mathrm{mSv}$ (for a chest x-ray study containing both posterior-anterior and lateral projections) (44). The effective doses based on using the DLP to an effective dose conversion coefficient in our study $(0.15-2.76 \mathrm{mSv})$ were somewhat higher than those reported on previously published LD chest CT studies. We used the conversion coefficient determined in this study $(k=0.024 \mathrm{mSv} / \mathrm{mGy} \cdot \mathrm{cm})$, which was higher than that of the frequently used conversion coefficients that vary between $0.014 \mathrm{mSv} / \mathrm{mGy} \cdot \mathrm{cm}$ and $0.020 \mathrm{mSv} / \mathrm{mGy} \cdot \mathrm{cm}$ $(17,18,26,28,30,31)$. This finding is due to the differences in the phantom models and our female-only conversion coefficient was close to the sex-averaged value reported by Zhang et al. (45) for the ICRP 110 reference phantoms $(0.026 \mathrm{mSv} / \mathrm{mGy} \cdot \mathrm{cm})$.

Organ segmentation uncertainty is a contributing factor in dosimetric uncertainty. The effect depends on the organ size, shape, position, and the dose distribution within each organ volumetric neighborhood. Consequently, the relative uncertainty of the organ dose due to segmentation is potentially higher for the small focal organs located near the borders of the scan range; that is, relatively small translations of the segmentation will bring the organ within the scan range or outside the scan range, or change the organ irradiated fraction at the scan range edge. This finding, in turn, may cause relatively high dose variations for such organs where the interquartile ranges of the voxel dose maps calculated over the organ volume are potentially within the same order of magnitude as the mean dose (eg, for the colon). However, the organs within the scan range (and the most contributing to effective dose) presented lower relative dose variations. The interquartile ranges of the dose maps for such organs were considerably lower than the mean organ dose (eg, for the lung). The other uncertainty factors include spectral and bowtie modeling uncertainties (36), computed tomography dose index-calibration uncertainty $(46,47)$, modeling of the $\mathrm{mA}$-modulation and tube start angle, and $\mathrm{MC}$ simulation statistical uncertainty; these factors provided a combined 15\% uncertainty. The effective dose uncertainty was not separately estimated because of the lack of standardized uncertainties for the tissue weighting factors and because the effective doses were used only for comparative purposes while using the same anthropomorphic model. However, the effective dose uncertainty would be higher than the average organ dose uncertainty.

Previous studies have discussed the effect of image quality on the confidence level of diagnosing pulmonary nodules in chest CT, particularly the detection of small nodules $(<5 \mathrm{~mm})(15,18-20,26,30,31)$. Our image quality results showed that the MBIR algorithm has significantly less image noise than that from images produced by conventional FBP and ASIR reconstructions. The images scanned with the LD 1 protocol and reconstructed with the MBIR algorithm presented an image noise level that was equal or smaller than the noise in standard-dose images reconstructed with either the ASIR or the FBP algorithm. Moreover, the edge spreading was typically lower in the MBIR images than that in the ASIR or the FBP reconstruction. However, the edge spreading in the MBIR images was inferior compared to the FBP and ASIR images in air plugs (simulating cavity-like metastases, small cysts, or centrilobular emphysemas) vs lung tissue boundaries. This observation may support previous findings of inferior detection of small ground-glass nodules in the lungs with MBIR $(17,30,31)$. Furthermore, the regularization in MBIR may result in highly nonstationary noise in the images, specifically at the contrast edges (34). This may also explain the inferior MBIR results concerning air plug vs lung tissue edge-spread function. Based on our results, the LD chest CT examinations with MBIR maintained the image 
contrast at comparable levels, although some variation in the lung tissues was observed between the reconstructions. Additionally, a confounding factor may arise from the lower mean energy of the $\mathrm{x}$-ray spectra and greater attenuation, particularly in bone tissue that used the $100-\mathrm{kVp}$ tube voltage protocols than in the $120-\mathrm{kVp}$ tube voltage protocols.

Our results support previous findings that lower image noise can be achieved with the MBIR algorithms compared to FBP and statistical iterative reconstruction algorithms, which support greater dose reduction possibilities and improved visibilities of small anatomic structures in the chest (subpleural vessels, lung fissures, and other small structures) $(16,22,27)$. Additionally, MBIR algorithms can reduce streak artifacts that can interfere with adequate visualization of small and low-contrast lesions in the lungs (12). Neroladaki et al. (17) found that it was possible to reduce patient radiation exposure significantly when detecting noncalcified pulmonary nodules. However, for characterizing ground-glass opacities and emphysemas, LD levels are clearly inferior to standard-dose levels. Similarly, Huber et al. (30) reported a drop of sensitivity for the detection of small $(5 \mathrm{~mm})$ groundglass nodules. According to their results, however, the smallest ground-glass nodules were still better visualized than the comparatively sized solid nodules. Although the edge spreading was higher in air plug vs lung tissue boundaries with MBIR compared to other reconstructions, we did not encounter any problems in visualizing the $5-\mathrm{mm}$ solid nodules or the 5-mm cavity-like metastases (or cysts or centrilobular emphysemas) in the lungs in our anthropomorphic phantom study. The comparability of air plug-in-lung-resolution measurement was hindered by the relatively high noise content in the FBP and ASIR images. MBIR nonlinearities at certain contrast levels and tissue boundaries may also have been an explanatory factor for the observed difference.

Our radiation dose and image quality findings are consistent with other LD and LD chest CT studies in different indications. These studies have also shown significant dose reductions $(27.0-98.6 \%)$ with several iterative reconstruction techniques. In these studies, MBIR algorithms yielded higher dose reductions than those observed in statistical iterative reconstruction methods; image quality was still maintained at an acceptable diagnostic level (12,15-23,26-31). However, it should be noted that the dose reduction percentages depend strongly on the radiation dose levels used in the standard-dose protocols.

There are limitations in using anthropomorphic phantoms to study the required image quality in clinical situations with various patients. Specifically, the lung structures of the anthropomorphic phantom in our study were rather simplistic, and we only used soft tissue plugs and three air plugs to simulate lung metastases. Thus, the simulated targets and boundaries represented only a few sites and a limited selection of Hounsfield unit values. The shape, contrast, and size of these simulated metastases should be varied in future investigations. However, because of the geometric and attenuation properties of the round-shaped lung metastases seen in patients with sarcoma, remarkably lower doses compared to standard chest CT protocols may be justified. Despite this possibility, the results of every phantom study in clinical situations should be viewed with caution, as the use of anthropomorphic phantoms for evaluating clinical image quality is limited. This also means that the $0.16-\mathrm{mSv}$ effective dose level achieved in the present study may be too low for achieving adequate image quality for a chest $\mathrm{CT}$ that follows lung metastases in patients with sarcoma, especially when scanning obese patients. Therefore, clinical research with patients with soft tissue sarcoma is needed. As a second limitation, we did not determine organ doses or effective doses that resulted in scout imaging; we investigated only helical CT scans. To dosimetrically compare LD protocols to the standard chest CT examination, the radiation burden of the scout image should also be considered, as its relative proportion in LD CT examinations may be particularly high $(48,49)$. Thus, the dose reduction percentage values of the whole-chest CT examinations may be altered from those of the presented values. As a third limitation, only one CT scanner from a single vendor was used. The investigated MBIR algorithm is available in a few GE CT scanner models. As other CT vendors also offer MBIR algorithms for their newest CT models, an extensive performance comparison is highly warranted. The MBIR algorithm in the present study used a complex system of prediction models. The reconstruction time was therefore approximately 40 minutes per scan, potentially limiting its use in a clinical environment. However, as chest CT examinations for diagnosing lung metastases in patients with sarcoma are performed in elective patients, the reconstruction time of MBIR is not an obstacle unlike for emergency and urgent patients. Finally, we determined the organ doses as the average reading of a limited number of MOSFET point measurements, which caused uncertainties specifically for the tissues located partially outside of the primary scanning range. However, the MC simulation results support our findings.

\section{CONCLUSIONS}

Our phantom study results showed that the use of the LD chest CT protocol for detecting lung metastases in patients with sarcoma could potentially reduce organ and effective doses in helical scans up to $95 \%$ compared to that of our standard-dose chest CT protocol while maintaining image quality. The MC simulations were in agreement with the MOSFET measurements. The MBIR algorithm appeared to result in lower image noise levels and higher spatial resolution than the conventional FBP or ASIR algorithms.

\section{ACKNOWLEDGMENTS}

The authors thank Dr. Esa Tuominen for assistance in constructing ultra-low-dose chest CT protocols for the CT imaging of patients with sarcoma and the Radiation and Nuclear Safety Authority of Finland (STUK) for the possibility of using the ImpactMC software. 


\section{REFERENCES}

1. Billingsley KG, Burt ME, Jara E, et al. Pulmonary metastases from soft tissue sarcoma: analysis of patterns of diseases and postmetastasis survival. Ann Surg 1999; 229:602-610.

2. Cormier JN, Pollock RE. Soft tissue sarcomas. CA Cancer J Clin 2004; 54:94-109.

3. BEIR (Committee on the Biological Effects of lonizing Radiations). Health risks from exposure to low levels of ionizing radiation: BEIR VII-phase 2. Washington, DC: The National Academies Press, 2006 National Research Council of the National Academies.

4. Brenner DJ, Hall EJ. Computed tomography-an increasing source of radiation exposure. N Engl J Med 2007; 357:2277-2284.

5. Pearce MS, Salotti JA, Little MP, et al. Radiation exposure from CT scans in childhood and subsequent risk of leukaemia and brain tumours: a retrospective cohort study. Lancet 2012; 380:499-505.

6. Kalra MK, Maher MM, Toth TL, et al. Techniques and applications of automatic tube current modulation for CT. Radiology 2004; 233:649657.

7. Schueller-Weidekamm C, Schaefer-Prokop CM, Weber M, et al. CT angiography of pulmonary arteries to detect pulmonary embolism: improvement of vascular enhancement with low kilovoltage settings. Radiology 2006; 241:899-907.

8. Kalender WA, Buchenau S, Deak P, et al. Technical approaches to the optimisation of CT. Phys Med 2008; 24:71-79.

9. Deak PD, Langner $\mathrm{O}$, Lell $\mathrm{M}$, et al. Effects of adaptive section collimation on patient radiation dose in multisection spiral CT. Radiology 2009; 252:140-147

10. Wang J, Duan X, Christner JA, et al. Radiation dose reduction to the breast in thoracic CT: comparison of bismuth shielding, organ-based tube current modulation, and use of a globally decreased tube current. Med Phys 2011; 38:6084-6092.

11. Thibault JB, Sauer KD, Bouman CA, et al. A three-dimensional statistical approach to improve image quality for multislice helical CT. Med Phys 2007; 34:4526-4544.

12. Katsura M, Matsuda I, Akahane M, et al. Model-based iterative reconstruction technique for radiation dose reduction in chest CT: comparison with the adaptive statistical iterative reconstruction technique. Eur Radiol 2012; 22:1613-1623.

13. Pickhardt PJ, Lubner MG, Kim DH, et al. Abdominal CT with modelbased iterative reconstruction (MBIR): initial results of a prospective trial comparing ultralow-dose with standard-dose imaging. Am J Roentgenol 2012; 199:1266-1274.

14. Deák Z, Grimm JM, Treitl M. Filtered back projection, adaptive statistical iterative reconstruction, and a model-based iterative reconstruction in abdominal CT: an experimental clinical study. Radiology 2013; 266:197-206.

15. Katsura M, Matsuda I, Akahane M, et al. Model-based iterative reconstruction technique for ultralow-dose chest CT: comparison of pulmonary nodule detectability with the adaptive statistical iterative reconstruction technique. Invest Radiol 2013; 48:206-212.

16. Miéville FA, Berteloot L, Grandjean A, et al. Model-based iterative reconstruction in pediatric chest CT: assessment of image quality in a prospective study of children with cystic fibrosis. Pediatr Radiol 2013; 43:558-567.

17. Neroladaki A, Botsikas D, Boudabbous S, et al. Computed tomography of the chest with model-based iterative reconstruction using a radiation exposure similar to chest X-ray examination: preliminary observations. Eur Radiol 2013; 23:360-366.

18. $\mathrm{Xu} \mathrm{Y,} \mathrm{He} \mathrm{W,} \mathrm{Chen} \mathrm{H}$, et al. Impact of the adaptive statistical iterative reconstruction technique on image quality in ultra-low-dose CT. Clin Radiol 2013; 68:902-908.

19. Gordic S, Morsbach F, Schmidt B, et al. Ultralow-dose chest computed tomography for pulmonary nodule detection. Invest Radiol 2014; 49:465-473.

20. Kim H, Park CM, Song YS, et al. Influence of radiation dose and iterative reconstruction algorithms for measurement accuracy and reproducibility of pulmonary nodule volumetry: a phantom study. Eur J Radiol 2014; 83:848-857.

21. Kim H, Park CM, Kim SH, et al. Persistent pulmonary subsolid nodules: model-based iterative reconstruction for nodule classification and measurement variability on low-dose CT. Eur Radiol 2014; 24:2700-2708.

22. Sun J, Peng $Y$, Duan $X$, et al. Image quality in children with low-radiation chest CT using adaptive statistical iterative reconstruction and modelbased iterative reconstruction. PLoS ONE 2014; 9. e96045.
23. Willemink MJ, Takx RAP, de Jong PA, et al. Computed tomography radiation dose reduction: effect of different iterative reconstruction algorithms on image quality. J Comput Assist Tomogr 2014; 38:815-823.

24. Hérin E, Gardavaud F, Chiaradia M, et al. Use of model-based iterative reconstruction (MBIR) in reduced-dose CT for routine follow-up of patients with malignant lymphoma: dose savings, image quality and phantom study. Eur Radiol 2015; 25:2362-2370.

25. Kaasalainen T, Palmu K, Lampinen A, et al. Limiting CT radiation dose in children with craniosynostosis: phantom study using model-based iterative reconstruction. Pediatr Radiol 2015; 45:1544-1553.

26. Rampinelli C, Origgi D, Vecchi V, et al. Ultra-low-dose CT with modelbased iterative reconstruction (MBIR): detection of ground-glass nodules in an anthropomorphic phantom study. Radiol Med 2015; 120:611-617.

27. Yuki H, Oda S, Utsunomiya D, et al. Clinical impact of model-based type iterative reconstruction with fast reconstruction time on image quality of low-dose screening chest CT. Acta Radiol 2016; 57:295-302.

28. Wang R, Sui X, Schoep UJ, et al. Ultralow-radiation dose chest CT: accuracy for lung densitometry and emphysema detection. Am J Roentgenol 2015; 204:743-749

29. Deák Z, Maertz F, Meurer F, et al. Submillisievert computed tomography of the chest using model-based iterative algorithm: optimization of tube voltage with regard to patient size. J Comput Assist Tomogr 2017; 41:254-262.

30. Huber A, Landau J, Ebner L, et al. Performance of ultralow-dose CT iterative reconstruction in lung cancer screening: limiting radiation dose exposure to the equivalent of conventional chest X-ray imaging. Eur Radiol 2016; 26:3643-3652.

31. Messerli M, Kluckert T, Knitel M, et al. Ultralow dose CT for pulmonary nodule detection with chest $\mathrm{x}$-ray equivalent dose-a prospective intraindividual comparative study. Eur Radiol 2017; 27:3290-3299.

32. Padole A, Singh S, Ackman JB, et al. Submillisievert chest CT with filtered back projection and iterative reconstruction techniques. Am J Roentgenol 2014; 203:772-781.

33. Padole A, Singh S, Lira D, et al. Assessment of filtered back projection, adaptive statistical, and model-based iterative reconstruction for reduced dose abdominal computed tomography. J Comput Assist Tomogr 2015; 39:462-467.

34. Solomon J, Samei E. Quantum noise properties of CT images with anatomical textured backgrounds across reconstruction algorithms: FBP and SAFIRE. Med Phys 2014; 41:091908.

35. Poludniowski G, Landry G, DeBlois F, et al. SpekCalc: a program to calculate photon spectra from tungsten anode x-ray tubes. Phys Med Biol 2009; 54:N433-N438.

36. Alikhani B, Büermann L. Non-invasive experimental determination of a CT source model. Phys Med 2016; 32:59-66.

37. International Commission on Radiological Protection (ICRP). Adult reference computational phantoms. ICRP Publication 110. Ann ICRP 2009; 39:1-164.

38. Lagarias JC, Reeds JA, Wright MH, et al. Convergence properties of the Nelder-Mead simplex method in low dimensions. SIAM J Optim 1998; 9:112-147.

39. Kaasalainen T, Palmu K, Lampinen A, et al. Effect of vertical positioning on organ dose, image noise and contrast in pediatric chest CT-phantom study. Pediatr Radiol 2013; 43:673-684.

40. Baek J, Pelc NJ. The noise power spectrum in CT with direct fan beam reconstruction. Med Phys 2010; 37:2074-2081.

41. Sanders J, Hurwitz L, Samei E. Patient-specific quantification of image quality: an automated method for measuring spatial resolution in clinical CT images. Med Phys 2016; 43:5330.

42. Otsu N. A threshold selection method from gray-level histograms. IEEE Trans Systems Man Cybern 1970; 9:62-66.

43. Suomalainen A, Kiljunen T, Käser Y, et al. Dosimetry and image quality of four dental cone beam computed tomography scanners compared with multislice computed tomography scanners. Dentomaxillofac Radiol 2009; 38:367-378.

44. Mettler FA, Huda W, Yoshizumi TT, et al. Effective doses in radiology and diagnostic nuclear medicine: a catalog. Radiology 2008; 248:254-263.

45. Zhang Y, Li X, Segars WP, et al. Organ doses, effective doses, and risk indices in adult CT: comparison of four types of reference phantoms across different examination protocols. Med Phys 2012; 39:3404-3423.

46. Deak P, van Straten M, Shrimpton PC, et al. Validation of a Monte Carlo tool for patient-specific dose simulations in multi-slice computed tomography. Eur Radiol 2008; 18:759-772.

47. Myronakis M, Perisinakis K, Tzedakis A, et al. Evaluation of a patientspecific Monte Carlo software for CT dosimetry. Radiat Prot Dosimetry 2009; 133:248-255 
48. Schmidt B, Saltybaeva N, Kolditz D, et al. Assessment of patient dose from CT localizer radiographs. Med Phys 2013; 40:084301.

49. Schmidt B, Hupfer M, Saltybaeva N, et al. Dose optimization for computed tomography localizer radiographs for low-dose lung computed tomography examinations. Invest Radiol 2017; 52:81-86.

\section{SUPPLEMENTARY DATA}

Supplementary data related to this article can be found at https://doi.org/10.1016/j.acra.2018.03.028. 